\title{
Erratum to: Angiomyolipoma being surgically excised for presumed kidney carcinoma
}

\author{
Weibin $\mathrm{Hou}^{1} \cdot \mathrm{He} \mathrm{Xiao}^{1} \cdot$ Guanghua Liu $^{1} \cdot$ Zhigang Ji $^{1}$
}

Published online: 17 December 2015

(C) Springer Science+Business Media Dordrecht 2015

\section{Erratum to: Int Urol Nephrol (2015) 47:1037-1043 DOI 10.1007/s11255-015-0996-y}

Unfortunately, some errors occurred in the original publication of the article. The authors apologize to the editors and readers for an error in the second sentence in the Materials and methods section of Abstract and Table 1. The corrected Abstract and the new version of Table 1 are given below.

\begin{abstract}
Materials and methods From 2008 to 2014, AML was pathologically confirmed in 38 patients who underwent
\end{abstract}

radical or partial nephrectomy for presumed malignant renal tumor. Control group 1 were patients with renal cell carcinoma (RCC) matched for sex and tumor size; control group 2 were patients with typical AML matched for age and sex. Pertinent data of the studied group and its matched control groups were recorded and analyzed.

The online version of the original article can be found under doi:10.1007/s11255-015-0996-y.

\section{Zhigang Ji}

jizg1129@163.com

1 Department of Urology, Peking Union Medical College Hospital, Chinese Academy of Medical Sciences and Peking

Union Medical College, 1\#, Shuaifuyuan, Dongcheng

District, Beijing 100730, People's Republic of China 
Table 1 Comparison of misdiagnosed AML group and its matched RCC control group

\begin{tabular}{|c|c|c|c|c|}
\hline Parameter & All patients $(n=76)$ & AMLs $(n=38)$ & $\operatorname{RCCs}(n=38)$ & $P$ \\
\hline Sex & & & & 1 \\
\hline Male & 34 & 17 & 17 & \\
\hline Female & 42 & 21 & 21 & \\
\hline Tumor size $(\mathrm{cm})$ & & & & 0.930 \\
\hline Mean \pm SD & $3.14 \pm 1.68$ & $3.12 \pm 1.68$ & $3.16 \pm 1.70$ & \\
\hline Range & $0.9-9.6$ & $0.9-9.4$ & $0.9-9.6$ & \\
\hline Age (years) & & & & 0.001 \\
\hline Mean $\pm \mathrm{SD}$ & $52.54 \pm 12.41$ & $48.11 \pm 12.92$ & $56.97 \pm 10.23$ & \\
\hline Range & $20-80$ & $20-74$ & $31-80$ & \\
\hline Laterality & & & & 0.818 \\
\hline Left & 39 & 19 & 20 & \\
\hline Right & 37 & 19 & 18 & \\
\hline Presentation & & & & 1 \\
\hline Incidental & 6 & 3 & 3 & \\
\hline Symptomatic & 70 & 35 & 35 & \\
\hline Ultrasonography & & & & 0.526 \\
\hline Hypoechoic & 50 & 24 & 26 & \\
\hline Isoechoic & 10 & 4 & 6 & \\
\hline Hyperechoic & 11 & 7 & 4 & \\
\hline Fat content on CT & & & & 0.077 \\
\hline Yes & 3 & 3 & 0 & \\
\hline No & 73 & 35 & 38 & \\
\hline Nephrectomy type & & & & 1 \\
\hline Radical & 16 & 8 & 8 & \\
\hline Partial & 60 & 30 & 30 & \\
\hline Surgical approach & & & & 0.238 \\
\hline Open & 29 & 17 & 12 & \\
\hline Laparoscopy & 47 & 21 & 26 & \\
\hline
\end{tabular}

